\title{
Interaction of Hawking radiation with static sources in de Sitter and Schwarzschild-de Sitter spacetimes
}

\author{
J. Castiñeiras, ${ }^{*}$ I. P. Costa e Silva, ${ }^{\dagger}$ and G. E. A. Matsas ${ }^{\ddagger}$ \\ Instituto de Física Teórica, Universidade Estadual Paulista, Rua Pamplona 145, 01405-900 São Paulo, São Paulo, Brazil
}

(Received 19 July 2003; published 31 October 2003)

\begin{abstract}
We study and look for similarities between the response rates $R^{\mathrm{dS}}\left(a_{0}, \Lambda\right)$ and $R^{\mathrm{SdS}}\left(a_{0}, \Lambda, M\right)$ of a static scalar source with constant proper acceleration $a_{0}$ interacting with a massless, conformally coupled KleinGordon field (i) in de Sitter spacetime, in the Euclidean vacuum, which describes a thermal flux of radiation emanating from the de Sitter cosmological horizon and (ii) in Schwarzschild-de Sitter spacetime, in the Gibbons-Hawking vacuum, which describes thermal fluxes of radiation emanating from both the hole and the cosmological horizons, respectively, where $\Lambda$ is the cosmological constant and $M$ is the black hole mass. After performing the field quantization in each of the above spacetimes, we obtain the response rates at the tree level in terms of an infinite sum of zero-energy field modes possessing all possible angular momentum quantum numbers. In the case of de Sitter spacetime, this formula is worked out and a closed, analytical form is obtained. In the case of Schwarzschild-de Sitter spacetime such a closed formula could not be obtained, and a numerical analysis is performed. We conclude, in particular, that $R^{\mathrm{dS}}\left(a_{0}, \Lambda\right)$ and $R^{\mathrm{SdS}}\left(a_{0}, \Lambda, M\right)$ do not coincide in general, but tend to each other when $\Lambda \rightarrow 0$ or $a_{0} \rightarrow \infty$. Our results are also contrasted and shown to agree (in the proper limits) with related ones in the literature.
\end{abstract}

DOI: $10.1103 /$ PhysRevD.68.084022

PACS number(s): 04.70.Dy, 04.62.+v

\section{INTRODUCTION}

It is well known from classical electrodynamics that accelerated electric charges radiate as seen by an inertial observer in Minkowski spacetime. However, according to the equivalence principle, a uniformly accelerated charge is seen by a comoving observer as being static in a "uniform gravitational field," and thus it is not expected to radiate. In the classical context, this apparent paradox was worked out in some detail by Rohrlich, Fulton [1], and Boulware [2].

The same problem has also been analyzed in a quantum context [3], in terms of photon emission rates, using the fact that an observer comoving with a uniformly accelerated charge views the latter as immersed in a Fulling-DaviesUnruh (FDU) thermal bath [4,5]. More specifically, the interaction of the static charge (as computed by comoving observers) with the FDU thermal bath results in the absorption and stimulated emission of zero-energy Rindler photons, which, although unobservable, nevertheless exactly account for the usual photon emission described by an inertial observer.

A particularly interesting arena to study interactions between sources and radiation is the vicinity of black holes, where the presence of nontrivial classical and quantum effects offers a wealth of conceptual and technical challenges. In this setting, it has recently been shown that the response $R^{\text {Sch }}\left(a_{0}, M\right)$ of a pointlike static scalar source with proper acceleration $a_{0}$ outside a Schwarzschild black hole of mass $M$ interacting with massless scalar particles of Hawking ra-

\footnotetext{
*Electronic address: jcastin@ift.unesp.br

†Electronic address: ivanpcs@ift.unesp.br

‡Electronic address: matsas@ift.unesp.br
}

diation (associated with the Unruh vacuum) is exactly the same as the response $R^{\mathrm{M}}\left(a_{0}\right) \equiv q^{2} a_{0} / 4 \pi^{2}$ (in natural units, where $q$ is a small coupling constant) of such a source when it is uniformly accelerated with the same proper acceleration in the inertial vacuum of Minkowski spacetime or, equivalently, when it is static in the FDU thermal bath of the Rindler spacetime [6]. This is surprising because structureless static scalar sources can only interact with zero-energy field modes; such modes probe the global geometry of spacetime and are accordingly quite different in Schwarzschild and Rindler spacetimes. Indeed, this equivalence is not verified, e.g., when either (i) the Unruh vacuum is replaced by the Hartle-Hawking vacuum [6], (ii) the black hole is endowed with electric charge [7] or (iii) the massless Klein-Gordon field is replaced with electromagnetic [8] or massive KleinGordon [9] ones. It is hitherto unclear whether the equivalence found in Ref. [6] is only a remarkable coincidence or if there is something deeper behind it. This circumstance has motivated us to study whether or not the equivalence would persist when one includes the presence of a cosmological constant, i.e., by replacing Schwarzschild with Schwarzschild-de Sitter (SdS) spacetime and Minkowski with de Sitter spacetime.

SdS spacetime may be viewed as describing a spherically symmetric black hole immersed in a universe with a positive cosmological constant $\Lambda>0$. It has attracted much attention lately on account of recent type Ia supernovae and cosmic microwave background observations [10] indicating that the Universe at large scale has (approximately) flat spatial geometry and is in accelerated expansion. These data suggest the existence of some background form of energy ("dark energy") with negative pressure. The most plausible scenarios to describe this energy include the existence of a positive cosmological constant and quintessence fields. Although in the latter case the energy density of the dark energy is allowed to change in time, in many models this variation can 
be neglected for astrophysically relevant scales. In other words, when considering objects such as black holes, one can for most purposes assume the presence of an effective, positive cosmological constant. In light of these facts, the interest in considering SdS black holes becomes clear.

In this paper we consider a static, pointlike source interacting with a conformally coupled, massless Klein-Gordon field in both de Sitter and SdS spacetimes. Quantum field theory in de Sitter spacetime has been much studied in the literature [11]. In this case, we take the field state to be the Euclidean vacuum (see, e.g., Ref. [12]) describing a thermal bath as seen by static observers. Our calculations in de Sitter closely follow those of Higuchi [13], but our presentation is somewhat different. In particular, it is useful for our purposes to derive the response rate of our pointlike source at a generic position inside the cosmological radius. In the $\mathrm{SdS}$ case, there are two main technical hindrances in considering the quantization of the Klein-Gordon field. The first one is the definition of what we shall call the Gibbons-Hawking vacuum [14]. This state describes a situation in which we have thermal fluxes emanating from both the hole and the cosmological event horizons. As usually, the related temperatures are proportional to the corresponding surface gravities $\kappa_{h}$ and $\kappa_{c}$. Because in general $\kappa_{h} \neq \kappa_{c}$, there are technical difficulties in defining such a state $[14,15]$ in the whole SdS spacetime. However, for the region between the horizons one may devise an heuristic prescription to define it, since in realistic situations where black holes are formed by gravitational collapse in a de Sitter background, it is natural to expect the emission of thermal radiation from both horizons (see [14] for an outline and further justification for the mentioned prescription). We shall not dwell on these problems in this paper but simply assume that radiation emanates from both horizons at definite temperatures. The second technical difficulty is related with the quantization of the scalar field in $\mathrm{SdS}$ spacetime. Due to the spherical symmetry of the problem, the corresponding Klein-Gordon equation is easily separated, but its radial part, except for the near extremal case [16], does not appear to be amenable to analytical treatment. Accordingly, we shall proceed to its numerical resolution.

The paper is organized as follows. In Sec. II, we briefly review some geometrical aspects of SdS and de Sitter spacetimes which will be useful for establishing the setting for our analysis and fixing notation. In Sec. III we present the general formalism to quantize a massless, conformally coupled scalar field in the background of interest. In Sec. IV we apply the formalism to de Sitter spacetime with the Euclidean vacuum, obtaining a simple, closed, analytical form for the response at the tree level of a static source interacting with the radiation from the cosmological horizon. In Sec. V, we apply the formalism to SdS spacetime with the GibbonsHawking vacuum and express the response of the static source in terms of a sum over the normal mode angular momenta, which is numerically evaluated. We then compare the behavior of this response with the one obtained in the de Sitter case (and with the related one obtained in Schwarzschild spacetime with the Unruh vacuum [6]). Finally, in Sec. VI we finish with some conclusions. Throughout this paper,

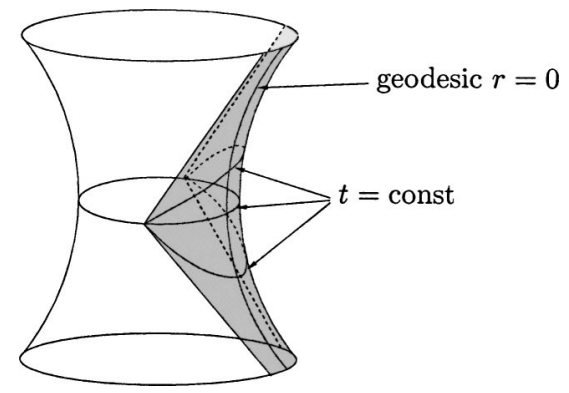

FIG. 1. Embedding of de Sitter spacetime in a flat background with two dimensions omitted (circular cross sections are to be thought of as copies of $S^{3}$ ). The shaded part represents the region of de Sitter spacetime covered by the coordinates $(t, r, \theta, \phi)$. One can pick any normal, timelike geodesic as the origin $r=0$.

we adopt natural units $\left(c=G=\hbar=k_{B}=1\right)$, the abstract index notation [17] and spacetime signature (+-- ).

\section{THE BACKGROUNDS: de SITTER AND SCHWARZSCHILD-de SITTER}

Before starting our central discussion, it will be useful to recall some geometrical features of de Sitter and SdS spacetimes. We shall briefly do so here, confining ourselves to the minimum of information necessary to our ends. For more details, see, e.g., Refs. [14,18].

de Sitter and SdS spacetimes are vacuum solutions of Einstein's field equations with a positive cosmological constant $\Lambda>0$. Their line elements can be written as

$$
d s^{2}=f(r) d t^{2}-f(r)^{-1} d r^{2}-r^{2}\left(d \theta^{2}+\sin ^{2} \theta d \phi^{2}\right),
$$

with $f(r) \mapsto f_{\mathrm{dS}}(r)=1-\Lambda r^{2} / 3$ for de Sitter spacetime and $f(r) \mapsto f_{\mathrm{SdS}}(r)=1-2 M / r-\Lambda r^{2} / 3$ for SdS spacetime [19], where $M$ denotes the mass of the corresponding black hole. Here, the "time coordinate" $t$ and the "angular coordinates" $\theta$ and $\phi$ have their usual ranges, $-\infty<t<+\infty, 0 \leqslant \theta<\pi$, $0 \leqslant \phi<2 \pi$, and for our purposes the "radial coordinate" $r$ must be restricted to non-negative values for which $f(r)$ $>0$.

Let us first consider de Sitter spacetime. We begin by defining the de Sitter or cosmological radius at $\alpha \equiv \sqrt{3 / \Lambda}$, and by noting that $f(r)>0$ implies $0 \leqslant r<\alpha$. The "singularity" at $r=\alpha$ is merely due to a bad choice of coordinates, and with an appropriate reparametrization [18] one can obtain the corresponding maximal analytic extension. This spacetime has topology $S^{3} \times \mathbb{R}$ and can be isometrically embedded as a one-sheeted hyperboloid in 5-dimensional Minkowski spacetime (see Fig. 1). The coordinates $(t, r, \theta, \phi)$ cover only part of de Sitter spacetime. The causal structure of de Sitter spacetime can be more readily visualized through the Penrose diagram in Fig. 2. The origin of the polar coordinates, $r=0$, and past and future infinities $\mathcal{I}^{-}$ and $\mathcal{I}^{+}$are represented by vertical and horizontal borderlines, respectively. We note that the region labeled as I in Fig. 2 [covered by the coordinates $(t, r, \theta, \phi)]$ on which we will focus has a global timelike future-directed Killing field $\xi^{a} \equiv(\partial / \partial t)^{a}$. The Killing field $\xi^{a}$ becomes lightlike at $r$ 


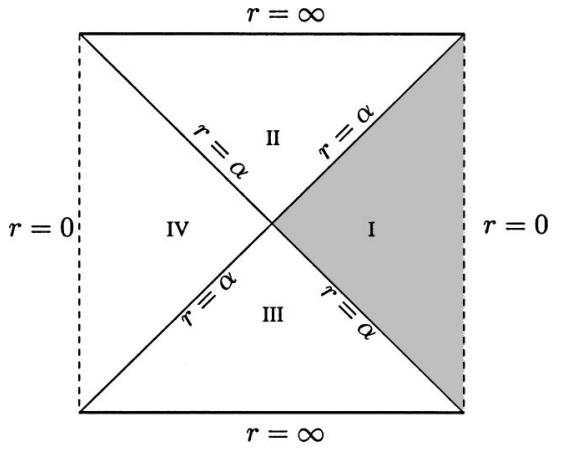

FIG. 2. Penrose diagram of de Sitter spacetime. The shaded region is the one covered by the coordinates $(t, r, \theta, \phi)$. Horizontal lines cutting this diagram represent 3 spheres, and the lines labeled as $r=0$ represent the worldlines of the "north and south pole" of these 3 spheres. The solid lines labeled as $r=\infty$ correspond to past and future infinities $\mathcal{I}^{-}$and $\mathcal{I}^{+}$.

$=\alpha$, which comprises a bifurcate Killing horizon (see, e.g., Ref. [15] for a definition). The observers following integral curves of the Killing field $\xi^{a}$ in region I will be called static for short. Static observers have 4-velocity $u^{a}$ $=\left(\xi^{c} \xi_{c}\right)^{-1 / 2} \xi^{a}, 4$-acceleration

$$
a^{a}=u^{b} \nabla_{b} u^{a} \equiv-\frac{r}{\alpha^{2}}\left(\frac{\partial}{\partial r}\right)^{a}
$$

and proper acceleration

$$
a_{\mathrm{dS}}=\sqrt{-a^{a} a_{a}}=\frac{r}{\alpha^{2}}\left(1-\frac{r^{2}}{\alpha^{2}}\right)^{-1 / 2} .
$$

It is thus clear that a static observer at $r=0$ follows indeed a geodesic.

Let us now turn our attention to SdS spacetime. We shall assume that $M / \alpha<1 / \sqrt{27}$. The zeroes of $f_{\mathrm{SdS}}(r)$ are, then, found at

$$
\begin{aligned}
& r_{c}=\frac{2 \alpha}{\sqrt{3}} \cos \left(\frac{A}{3}\right), \\
& r_{h}=\frac{-2 \alpha}{\sqrt{3}} \cos \left(\frac{A+\pi}{3}\right), \\
& r_{3}=-\left(r_{c}+r_{h}\right),
\end{aligned}
$$

where $A \equiv \arccos \left[-\left(27 M^{2} / \alpha^{2}\right)^{1 / 2}\right]$ satisfies $\pi / 2<A<\pi$. Here, $r_{c}$ and $r_{h}$ are associated with the cosmological and black hole horizons, respectively, and satisfy $0<r_{h}<r_{c}$. Moreover, $f_{\mathrm{SdS}}(r)>0$ for $r_{h}<r<r_{c}$. The causal structure of the SdS spacetime is clear in the Penrose diagram [14] displayed in Fig. 3. We will be interested in the region I where $\xi^{a}=(\partial / \partial t)^{a}$ is a global timelike future-directed Killing field. In SdS spacetime, static observers have 4-velocity $u^{a}$ $=\left(\xi^{c} \xi_{c}\right)^{-1 / 2} \xi^{a}, 4$-acceleration

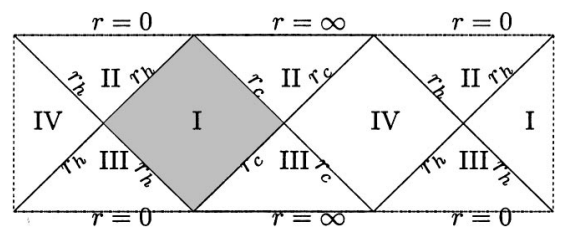

FIG. 3. Penrose Diagram of Schwarzschild-de Sitter spacetime. The displayed pattern repeats itself infinitely both to the left and to the right. The shaded region is a static, globally hyperbolic region by itself.

$$
a^{a}=u^{b} \nabla_{b} u^{a} \equiv\left(\frac{M}{r^{2}}-\frac{r}{\alpha^{2}}\right)\left(\frac{\partial}{\partial r}\right)^{a},
$$

and proper acceleration

$$
a_{\mathrm{SdS}}=\sqrt{-a^{a} a_{a}}=\left|\frac{M}{r^{2}}-\frac{r}{\alpha^{2}}\right|\left(1-\frac{2 M}{r}-\frac{r^{2}}{\alpha^{2}}\right)^{-1 / 2} .
$$

Note that static observers with $r=\left(M \alpha^{2}\right)^{1 / 3}$ follow geodesics $\left(a_{\mathrm{SdS}}=0\right)$, due to a balance between the cosmic repulsion and the black hole attraction.

\section{RESPONSE OF A STATIC SOURCE INTERACTING WITH A SCALAR FIELD}

Consider now the quantization of a massless, conformally coupled Klein-Gordon field $\Phi\left(x^{\mu}\right)$, in the background defined by Eq. (1), described by the action

$$
S=\frac{1}{2} \int d^{4} x \sqrt{-g}\left[\nabla^{a} \Phi \nabla_{a} \Phi-(1 / 6) R \Phi\right],
$$

where $g \equiv \operatorname{det}\left\{g_{a b}\right\}$, and $R=4 \Lambda=12 / \alpha^{2}$ is the scalar curvature for both de Sitter and SdS spacetimes. The associated Klein-Gordon equation is

$$
\nabla^{a} \nabla_{a} \Phi+(1 / 6) R \Phi=0 .
$$

It is well known that quantum field theory takes a relatively simple form in globally hyperbolic, stationary spacetimes where, in particular, a well defined notion of particle can be given (see, e.g., Ref. [20], and references therein). This is the case for the shaded regions in Figs. $2(0 \leqslant r$ $<\alpha)$ and $3\left(r_{h}<r<r_{c}\right)$. For each such region, we shall look for a set of positive-frequency modes

$$
u_{\omega l m}^{i}\left(x^{\mu}\right)=\sqrt{\frac{\omega}{\pi}} \frac{\psi_{\omega l}^{i}(r)}{r} Y_{l m}(\theta, \phi) e^{-i \omega t}
$$

associated with the timelike Killing field $\xi^{a}=(\partial / \partial t)^{a}$, where $\omega \geqslant 0, l \in \mathbb{Z}^{+}$and $m \in[-l, l] \cap \mathbb{Z}$ are the frequency and the angular momentum quantum numbers, respectively, and $Y_{l m}(\theta, \phi)$ are the spherical harmonics. The factor $\sqrt{\omega / \pi}$ has been introduced for later convenience. The radial part of Eq. (10) then reads 


$$
\left[-f(r) \frac{d}{d r}\left(f(r) \frac{d}{d r}\right)+V_{\mathrm{eff}}(r)\right] \psi_{\omega l}^{i}(r)=\omega^{2} \psi_{\omega l}^{i}(r),
$$

where the effective scattering potential $V_{\text {eff }}(r)$ is given by

$$
V_{\mathrm{eff}}(r)=f(r)\left(\frac{1}{r} \frac{d f}{d r}+\frac{l(l+1)}{r^{2}}+\frac{2}{\alpha^{2}}\right) .
$$

Note that Eq. (12) admits, in general, two sets of independent solutions which will be labeled with $i=\mathrm{I}$, II. The $u_{\omega l m}^{i}\left(x^{\mu}\right)$ modes are assumed to be orthonormalized with respect to the Klein-Gordon inner product [12]:

$$
\begin{aligned}
& i \int_{\Sigma} d \sum n^{a}\left(u_{\omega l m}^{i} * \nabla_{a} u_{\omega^{\prime} l^{\prime} m^{\prime}}^{i^{\prime}}-u_{\omega^{\prime} l^{\prime} m^{\prime}}^{i^{\prime}} \nabla_{a} u_{\omega l m}^{i} *\right) \\
& \quad=\delta_{i i^{\prime}} \delta_{l l^{\prime}} \delta_{m m^{\prime}} \delta\left(\omega-\omega^{\prime}\right), \\
& i \int_{\Sigma} d \sum n^{a}\left(u_{\omega l m}^{i} \nabla_{a} u_{\omega^{\prime} l^{\prime} m^{\prime}}^{i^{\prime}}-u_{\omega^{\prime} l^{\prime} m^{\prime}}^{i^{\prime}}, \nabla_{a} u_{\omega l m}^{i}\right)=0,
\end{aligned}
$$

where $n^{a}$ is the future-directed unit vector normal to some fixed Cauchy surface $\Sigma$. These modes and their respective complex conjugates form a complete orthonormal basis of the space of solutions of Eq. (10) in the regions of interest. As a result, we can expand the field operator as

$$
\hat{\Phi}\left(x^{\mu}\right)=\sum_{i=1, \mathrm{II}} \sum_{l=0}^{\infty} \sum_{m=-l}^{l} \int_{0}^{+\infty} d \omega\left[u_{\omega l m}^{i}\left(x^{\mu}\right) a_{\omega l m}^{i}+\text { H.c. }\right],
$$

where $a_{\omega l m}^{i}$ and $a_{\omega l m}^{i}{ }^{\dagger}$ are annihilation and creation operators, respectively, and satisfy the usual commutation relations

$$
\left[a_{\omega l m}^{i}, a_{\omega^{\prime} l^{\prime} m^{\prime}}^{i^{\prime}}\right]=\delta_{i i^{\prime}} \delta_{l l^{\prime}} \delta_{m m^{\prime}} \delta\left(\omega-\omega^{\prime}\right) .
$$

The "Boulware" vacuum $|0\rangle$ is defined by $a_{\omega l m}^{i}|0\rangle=0$ for every $i, \omega, l$ and $m$. This is the state of "no particles" as defined by the static observers following integral curves of $\xi^{a}$.

Let us consider now a pointlike static scalar source lying at $\left(r_{0}, \theta_{0}, \varphi_{0}\right)$ described by

$$
j\left(x^{\mu}\right)=(q / \sqrt{-h}) \delta\left(r-r_{0}\right) \delta\left(\theta-\theta_{0}\right) \delta\left(\varphi-\varphi_{0}\right),
$$

where $h=-f^{-1} r^{4} \sin ^{2} \theta$ is the determinant of the spatial metric induced on an equal $t$-time hypersurface $\Sigma$ and $q$ is a small coupling constant. This source is coupled to the KleinGordon field $\hat{\Phi}\left(x^{\mu}\right)$ via the interaction action

$$
\hat{S}_{\mathrm{I}}=\int d^{4} x \sqrt{-g} j \hat{\Phi} .
$$

All the calculations will be carried out at the tree level.

The total response, i.e., combined particle emission and absorption probabilities per unit proper time of the source, is given by

$$
R \equiv \sum_{i=1, \mathrm{II}} \sum_{l=0}^{\infty} \sum_{m=-l}^{l} \int_{0}^{+\infty} d \omega R_{\omega l m}^{i}
$$

where

$$
R_{\omega l m}^{i} \equiv \tau^{-1}\left\{\left|\mathcal{A}_{\omega l m}^{i}{ }^{\mathrm{em}}\right|^{2}\left[1+n^{i}(\omega)\right]+\left|\mathcal{A}_{\omega l m}^{i}{ }^{\mathrm{abs}}\right|^{2} n^{i}(\omega)\right\}
$$

and $\tau$ is the total proper time of the source. Here $\mathcal{A}_{\omega l m}^{i}$ em $\equiv\left\langle i \omega l m\left|\hat{S}_{\mathrm{I}}\right| 0\right\rangle$ and $\mathcal{A}_{\omega l m}^{i}{ }^{\text {abs }} \equiv\left\langle 0\left|\hat{S}_{\mathrm{I}}\right| i \omega l m\right\rangle$ are the emission and absorption amplitudes, respectively, of Boulware states $|i \omega l m\rangle$, and the $n^{i}$ factors depend on the field state chosen in each case.

Structureless static sources described by Eq. (17) can only interact with zero-energy modes [3] and thus the response of the source in the Boulware vacuum vanishes. However, in the presence of a background thermal bath, the absorption and stimulated emission rates lead to a non-zero response. In order to deal with zero-energy modes, we need a "regulator" to avoid the appearance of intermediate indefinite results (for a more comprehensive discussion on the interaction of static sources with zero-energy modes, see Ref. [3]). For this purpose, we let the coupling constant $q$ oscillate with frequency $\omega_{0}$ by replacing $q$ with $q_{\omega_{0}} \equiv \sqrt{2} q \cos \left(\omega_{0} t\right)$ in Eq. (17) and take the limit $\omega_{0} \rightarrow 0$ at the end of our calculations. The factor $\sqrt{2}$ has been introduced to ensure that the time average $\left\langle\left|q_{\omega_{0}}(t)\right|^{2}\right\rangle_{t}=q^{2}$ since the absorption and emission rates are functions of $q^{2}$. Another equivalent regularization procedure is discussed in [21]. A straightforward calculation using $\sum_{m=-l}^{l}\left|Y_{l m}\left(\theta_{0}, \varphi_{0}\right)\right|^{2}=(2 l+1) / 4 \pi[22]$ gives

$$
\begin{aligned}
R\left(r_{0}\right)= & \lim _{\omega_{0} \rightarrow 0} \sum_{i=\mathrm{I}, \mathrm{II}} \sum_{l=0}^{\infty} \frac{q^{2} \omega_{0} \sqrt{f\left(r_{0}\right)}}{4 \pi^{2} r_{0}^{2}} \\
& \times(2 l+1)\left|\psi_{\omega_{0} l}^{i}\left(r_{0}\right)\right|^{2}\left[1+2 n^{i}\left(\omega_{0}\right)\right] .
\end{aligned}
$$

\section{RESPONSE RATE IN de SITTER SPACETIME}

We are now ready to consider the response rate of the static source in de Sitter spacetime. Taking $f(r) \mapsto f_{\mathrm{dS}}(r)$ $\equiv\left(1-r^{2} / \alpha^{2}\right)$, the effective potential (13) becomes

$$
V_{\mathrm{eff}}^{\mathrm{dS}}(r)=\frac{l(l+1)}{r^{2}}\left(1-\frac{r^{2}}{\alpha^{2}}\right) .
$$

We define a new coordinate $z=\alpha / r$, and use it to reexpress Eq. (12), with potential (22), in the form

$$
\left[\frac{d}{d z}\left(\left(1-z^{2}\right) \frac{d}{d z}\right)+l(l+1)+\frac{\alpha^{2} \omega^{2}}{1-z^{2}}\right] \psi_{\omega l}^{i}=0,
$$

which is just the associated Legendre equation. It has two sets of linearly independent solutions, $P_{l}^{i \alpha \omega}(z)$ and $Q_{l}^{i \alpha \omega}(z)$ (cf., e.g., Ref. [22]), but only the latter is regular at $r=0$ $(z=\infty)$. Therefore we only consider modes of the form 


$$
u_{\omega l m}^{I \mathrm{dS}}\left(x^{\mu}\right)=C_{\omega l}^{\mathrm{I}} \sqrt{\frac{\omega}{\pi}} \frac{Q_{l}^{i \alpha \omega}(\alpha / r)}{r} Y_{l m}(\theta, \phi) e^{-i \omega t}
$$

where $C_{\omega l}^{\mathrm{I}}$ are normalization constants to be fixed by requiring that the modes be orthonormal with respect to the KleinGordon inner product (14). The physical behavior of the normal modes (24) is clear: one may visualize them in the shaded region of Fig. 2 as emanating from the past horizon, scattering off the line $r=0$, and being reflected back to the future horizon. Alternatively, one may think of the modes "in spatial terms" as converging from a 2-sphere at $r=\alpha$ onto its center at $r=0$ and then spreading out to $r=\alpha$ again. Of course, the modes "converge isotropically" only for $l$ $=0$, but "swirl around" as they plunge in onto $r=0$ for $l$ $\neq 0$.

Let us now evaluate the normalization constants $C_{\omega l}^{\mathrm{I}}$. For this purpose we substitute the modes (24) into Eq. (14), where we choose $\Sigma$ to be the $t=0$ hypersurface and we use the orthonormality of the spherical harmonics. We are then left with

$$
C_{\omega l}^{\mathrm{I}} * C_{\omega^{\prime} l}^{\mathrm{I}}(\alpha / \pi) \sqrt{\omega \omega^{\prime}}\left(\omega+\omega^{\prime}\right) I_{l \omega \omega^{\prime}}=\delta\left(\omega-\omega^{\prime}\right),
$$

where

$$
I_{l \omega \omega^{\prime}} \equiv \int_{0}^{1} \frac{d y}{1-y^{2}}\left[Q_{l}^{i \alpha \omega}(1 / y)\right]^{*} Q_{l}^{i \alpha \omega^{\prime}}(1 / y) .
$$

In order to evaluate $I_{l \omega \omega^{\prime}}$, we use formula 8.703 of Ref. [22] to write

$$
Q_{l}^{i \alpha \omega}(1 / y)=\frac{\sqrt{\pi} \Gamma(1+l+i \alpha \omega) y f_{\alpha \omega}^{l}(y)}{2^{l+1} \Gamma(l+3 / 2) e^{\alpha \omega \pi}},
$$

where we have defined

$$
\begin{aligned}
f_{\alpha \omega}^{l}(y) \equiv & y^{l}\left(1-y^{2}\right)^{i \alpha \omega / 2} \\
& \times F\left(\frac{2+l+i \alpha \omega}{2}, \frac{1+l+i \alpha \omega}{2} ; l+\frac{3}{2} ; y^{2}\right) .
\end{aligned}
$$

Here, $F(a, b ; c ; x)$ denotes a hypergeometric function. By using $f_{\alpha \omega}^{l}(y)$, Higuchi [13] evaluated the integral

$$
\begin{aligned}
I_{l \omega \omega^{\prime}}^{(2)}= & \int_{0}^{1} \frac{d y}{1-y^{2}} y^{2}\left[f_{\alpha \omega}^{l}(y)\right]^{*} f_{\alpha \omega^{\prime}}^{l}(y) \\
= & \frac{2 \pi}{\alpha}\left|\frac{\Gamma(l+3 / 2) \Gamma(i \alpha \omega)}{\Gamma[(2+l+i \alpha \omega) / 2] \Gamma[(1+l+i \alpha \omega) / 2]}\right|^{2} \\
& \times \delta\left(\omega-\omega^{\prime}\right) .
\end{aligned}
$$

We now use Eq. (29) to compute $I_{l \omega \omega^{\prime}}$ and substitute the result into Eq. (25). Apart from an unimportant global phase, we get

$$
C_{\omega l}^{I}=\frac{2^{l} e^{\alpha \omega \pi} \Gamma[(2+l+i \alpha \omega) / 2] \Gamma[(1+l+i \alpha \omega) / 2]}{\sqrt{\pi} \omega \Gamma(1+l+i \alpha \omega) \Gamma(i \alpha \omega)} .
$$

Next, we assume that the field is in the so-called "Euclidean" vacuum (also known as "Bunch-Davies" or "BirrellDavies" vacuum), which describes a thermal bath of temperature

$$
T_{\mathrm{dS}}=1 /(2 \pi \alpha)
$$

as measured by the inertial observer at $r=0$ (see, e.g., Refs. $[12,15,20]$, and references therein for further properties of this state). As a consequence, $n^{\mathrm{I}}(\omega) \equiv\left(e^{\omega \beta}-1\right)^{-1}$ with $\beta^{-1} \equiv T_{\mathrm{dS}}$.

In order to compute the response (21), we recall that in de Sitter spacetime the sum will be restricted to the set of regular modes, i.e., with $i=\mathrm{I}$. Then, we use $\psi_{\omega_{0} l}^{\mathrm{I}}\left(r_{0}\right)$ $\equiv C_{\omega_{0} l}^{\mathrm{I}} Q_{l}^{i \alpha \omega_{0}}\left(\alpha / r_{0}\right)$, where $C_{\omega_{0} l}^{\mathrm{I}}$ is obtained from Eq. (30), and the identity (cf. Eq. 8.332.1 in [22])

$$
|x \Gamma(i x)|^{2}=\pi x / \sinh (\pi x) .
$$

As a result, we obtain

$$
\begin{aligned}
R^{\mathrm{dS}}\left(r_{0}, \alpha\right)= & \frac{q^{2} \alpha f\left(r_{0}\right)^{1 / 2}}{4 \pi^{3} r_{0}^{2}} \sum_{l=0}^{\infty} 2^{2 l}(2 l+1) \\
& \times\left|\frac{\Gamma[(l+2) / 2] \Gamma[(l+1) / 2]}{\Gamma(l+1)}\right|^{2}\left|Q_{l}\left(\frac{\alpha}{r_{0}}\right)\right|^{2},
\end{aligned}
$$

where $Q_{l}(z)$ is the ordinary Legendre function. Now, we use the doubling formula [22]

$$
\Gamma(2 x)=2^{2 x-1} \pi^{-1 / 2} \Gamma(x) \Gamma(x+1 / 2),
$$

with $x \equiv(l+1) / 2$ in Eq. (32):

$$
R^{\mathrm{dS}}\left(r_{0}, \alpha\right)=\frac{q^{2} \alpha f\left(r_{0}\right)^{1 / 2}}{4 \pi^{2} r_{0}^{2}} \sum_{l=0}^{\infty}(2 l+1)\left|Q_{l}\left(\frac{\alpha}{r_{0}}\right)\right|^{2} .
$$

Finally, we use the identity [6]

$$
\sum_{l=0}^{\infty}\left|Q_{l}(s)\right|^{2}(2 l+1)=\frac{1}{s^{2}-1}
$$

in Eq. (33) to obtain the final response as a function of the source's position:

$$
R^{\mathrm{dS}}\left(r_{0}, \alpha\right)=\frac{q^{2}}{4 \pi^{2} \alpha}\left(1-\frac{r_{0}^{2}}{\alpha^{2}}\right)^{-1 / 2} .
$$

For $r_{0}=0$ we recover the formula for the response of an inertial source in de Sitter spacetime, given in Ref. [13]. It is convenient to invert Eq. (3) to write the response in terms of the source's proper acceleration, which is a coordinateindependent observable in general relativity: 


$$
R^{\mathrm{dS}}\left(a_{0}, \alpha\right)=\frac{q^{2}}{4 \pi^{2} \alpha}\left(1+\alpha^{2} a_{0}^{2}\right)^{1 / 2} .
$$

We note that when $\alpha a_{0} \gg 1$, i.e., when either the source approaches the cosmological horizon or the cosmological constant is small enough ( $\alpha$ being accordingly large), we have

$$
R^{\mathrm{dS}}\left(a_{0}, \alpha\right) \approx \frac{q^{2} a_{0}}{4 \pi^{2}} .
$$

The right-hand side of Eq. (37) is independent of $\alpha$ and acquires the same form as the response of a static source in the Rindler wedge (i.e., uniformly accelerated in Minkowski spacetime) interacting with a massless Klein-Gordon field in the usual inertial vacuum. To see why this occurs, let us first write from Eq. (3) the source's radial position as

$$
r_{0}\left(a_{0}, \alpha\right)=\alpha\left[1+\left(\alpha a_{0}\right)^{-2}\right]^{-1 / 2} .
$$

Thus, for $\alpha a_{0} \gg 1$, we obtain $r_{0} \approx \alpha$. Now, in this region, the de Sitter line element (1) reduces (apart from the angular piece) to the Rindler form

$$
d s_{\mathrm{dS}}^{2} \approx e^{2 \eta / \alpha} d t^{2}-e^{2 \eta / \alpha} d \eta^{2},
$$

where $\eta \equiv \alpha \ln \left(1-r^{2} / \alpha^{2}\right)^{1 / 2} \quad(-\infty<\eta \leqslant 0)$. Indeed, the proper distance between $r_{0}$ and the cosmological horizon is $\alpha \arctan \left[1 /\left(\alpha a_{0}\right)\right] \stackrel{\alpha a_{0} \rightarrow \infty}{\longrightarrow} 1 / a_{0}$, which is precisely the proper distance between a static source in the Rindler wedge with proper acceleration $a_{0}$ and its horizon. This observation combined with the fact that the local temperature at the source (obtained by multiplying temperature (31) by the Tolman factor [23]) corresponds to the temperature of the Fulling-Davies-Unruh thermal bath,

$$
T_{\mathrm{dS}}^{l o c}=\frac{T_{\mathrm{dS}}}{\sqrt{f_{\mathrm{dS}}\left(r_{0}\right)}} \approx \frac{a_{0}}{2 \pi},
$$

clarifies Eq. (37). In particular, the decrease in the temperature $T_{\mathrm{dS}}=1 /(2 \pi \alpha)$ [cf. Eq. (31)]; when $\alpha$ grows large is perfectly compensated by the source's approach to the horizon [cf. Eq. (38)].

\section{RESPONSE RATE IN SCHWARZSCHILD-de SITTER SPACETIME}

We now turn to the field quantization in SdS spacetime and compare the response rate calculated in this case with the one obtained in the previous section [see Eq. (36)]. Similarly, we shall select Klein-Gordon orthonormalized modes of the form (11). Equation (12) with the effective scattering potential for SdS spacetime

$$
V_{\mathrm{eff}}^{\mathrm{SdS}}(r)=\left(1-\frac{2 M}{r}-\frac{r^{2}}{\alpha^{2}}\right)\left(\frac{2 M}{r^{3}}+\frac{l(l+1)}{r^{2}}\right)
$$

admits, now, two sets of linearly independent regular solutions $\psi_{\omega l}^{i}$. We shall associate $\psi_{\omega l}^{\mathrm{I}} \equiv \psi_{\omega l}^{\overrightarrow{ }}$ and $\psi_{\omega l}^{\mathrm{II}} \equiv \psi_{\omega l}^{\leftarrow}$ with purely ingoing modes emanating from the white hole horizon
$\mathcal{H}_{h}^{-}$(hereafter simply referred as hole horizon) and from the past cosmological horizon $\mathcal{H}_{c}^{-}$, respectively. $\psi_{\omega l}^{\overrightarrow{b l}}$ and $\psi_{\omega l}^{\leftarrow}$ are Klein-Gordon orthogonal to each other, as can be seen by choosing the Cauchy surface in Eq. (14) to be $\Sigma$ $=\mathcal{H}_{h}^{-} \cup \mathcal{H}_{c}^{-}$, and then using the fact that $\psi_{\omega l}$ and $\psi_{\omega l}^{\leftarrow}$ vanish at $\mathcal{H}_{c}^{-}$and $\mathcal{H}_{h}^{-}$, respectively.

For the Gibbons-Hawking vacuum [14], the appropriate thermal factors appearing in Eq. (20) are $n^{\mathrm{I}}(\omega) \equiv\left(e^{\omega \beta_{h}}\right.$ $-1)^{-1}$ and $n^{\mathrm{II}}(\omega) \equiv\left(e^{\omega \beta_{c}}-1\right)^{-1}$, where

$$
\beta_{h}^{-1}=\frac{\kappa_{h}}{2 \pi} \text { and } \beta_{c}^{-1}=\frac{\kappa_{c}}{2 \pi}
$$

are the temperatures of the radiation from the hole and the cosmological horizons, respectively, with

$$
\begin{gathered}
\kappa_{h}=\left.\frac{1}{2} \frac{d f_{\mathrm{SdS}}}{d r}\right|_{r=r_{h}}=\frac{1}{2 \alpha^{2} r_{h}}\left(r_{c}-r_{h}\right)\left(r_{h}+\bar{r}\right) \\
\kappa_{c}=\left.\frac{1}{2} \frac{d f_{\mathrm{SdS}}}{d r}\right|_{r=r_{c}}=\frac{1}{2 \alpha^{2} r_{c}}\left(r_{c}-r_{h}\right)\left(r_{c}+\bar{r}\right)
\end{gathered}
$$

being the surface gravities of the corresponding horizons, where $\bar{r} \equiv r_{h}+r_{c}$. In this case, the response (21) can be written as

$$
\begin{aligned}
R^{\mathrm{SdS}}\left(r_{0}, M, \alpha\right)= & \frac{q^{2} \sqrt{f\left(r_{0}\right)}}{2 \pi r_{0}^{2}} \lim _{\omega_{0} \rightarrow 0} \sum_{i=\mathrm{I}, \mathrm{II}} \sum_{l=0}^{\infty}(2 l+1) \\
& \times \omega_{0}\left|\psi_{\omega_{0} l}^{i}\left(r_{0}\right)\right|^{2} n^{i}\left(\omega_{0}\right) .
\end{aligned}
$$

Equation (45) allows one to compute the response provided one has obtained the (normalized) modes. For numerical convenience, let us introduce a new coordinate

$$
x(r)=-\frac{1}{2 \kappa_{c}} \ln \left(1-\frac{r}{r_{c}}\right)+\frac{1}{2 \kappa_{h}} \ln \left(\frac{r}{r_{h}}-1\right)+\frac{1}{2 \bar{\kappa}} \ln \left(\frac{r}{\bar{r}}+1\right),
$$

where $\bar{\kappa} \equiv\left(2 \alpha^{2} \bar{r}\right)^{-1}\left(r_{c}+\bar{r}\right)\left(r_{h}+\bar{r}\right)$. In terms of the new variable $x$, Eq. (12) is recast in the "Schrödinger-like" form

$$
\left[-\frac{d^{2}}{d x^{2}}+V_{\mathrm{eff}}^{\mathrm{SdS}}[r(x)]\right] \psi_{\omega l}^{i}(x)=\omega^{2} \psi_{\omega l}^{i}(x) .
$$

Near the horizons [and assuming the realistic case $M \ll \alpha$ (i.e., $r_{h} \ll r_{c}$ )], we have

$$
V_{\text {eff }}^{\mathrm{SdS}}(x) \sim \begin{cases}e^{-2 \kappa_{c} x} \ll 1 & \text { for } x \gg \alpha \\ e^{2 \kappa_{h} x} \ll 1 & \text { for } x<0,|x| \gg 2 M .\end{cases}
$$

Thus, in these regions the potential becomes exponentially suppressed, and we can approximate Eq. (47) by

$$
-\frac{d^{2} \psi_{\omega l}^{i}}{d x^{2}} \approx \omega^{2} \psi_{\omega l}^{i}(x)
$$




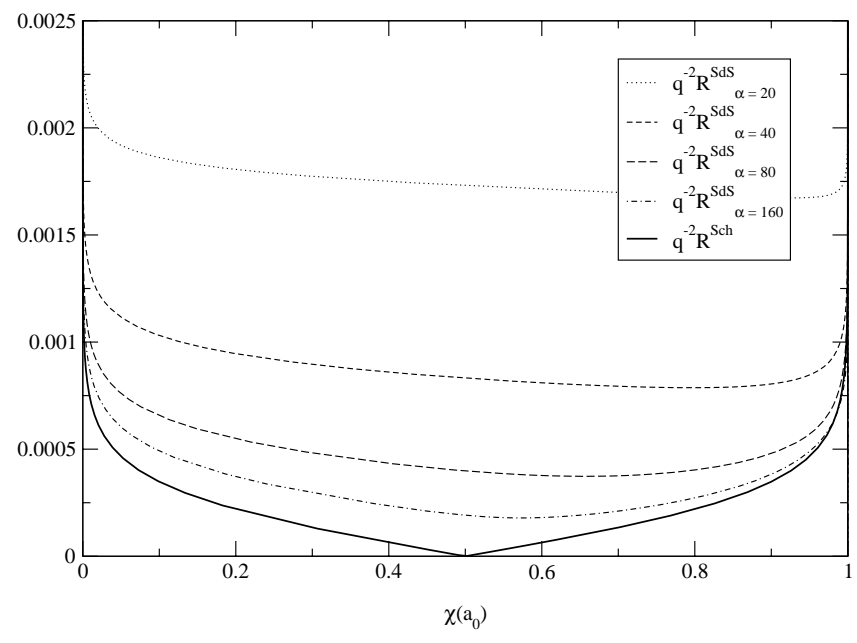

FIG. 4. $R^{\mathrm{SdS}}$ is plotted vs $\chi\left(a_{0}\right)$ (which is a monotonic function of $a_{0}$ ) for various values of $\alpha$ [with $M=2, \omega_{0}=10^{-4}, \rho=80$ and the sum over $l$ is performed up to $l=8$ (inclusive)]. In particular, $\chi\left(a_{0}\right)=0, \quad \chi\left(a_{0}\right)=1 / 2$, and $\chi\left(a_{0}\right)=1$ correspond to the cases where the source is at $r_{0}=r_{h}$, geodesic at $r_{0}=\left(M \alpha^{2}\right)^{1 / 3}$, and at $r_{0}=r_{c}$, respectively. Note that $R^{\mathrm{SdS}}$ approaches $R^{\mathrm{Sch}}$ (bottom graph) as $\alpha$ increases.

This leads to the asymptotic behavior of the modes

$$
\psi_{\omega l}(x) \approx \begin{cases}A_{\omega l}\left(e^{i \omega x}+\mathcal{R}_{\omega l} e^{-i \omega x}\right) & (x<0,|x| \gg 2 M) \\ A_{\omega l} \mathcal{T}_{\omega l} e^{i \omega x} & (x \gg \alpha)\end{cases}
$$

and

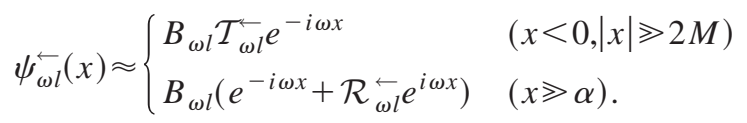

Here, $\left|\mathcal{R}_{\omega l}\right|^{2},\left|\mathcal{R}_{\omega l}^{\leftarrow}\right|^{2}$ and $\left|\mathcal{T}_{\omega l}\right|^{2},\left|\mathcal{T}_{\omega l}^{\leftarrow}\right|^{2}$ are reflection and transmission coefficients, respectively, for the "scattering problem" defined by Eq. (47). They satisfy usual "conservation of probability" laws: $\left|\mathcal{R}_{\omega l}^{\overrightarrow{\omega l}}\right|^{2}+\left|\mathcal{T}_{\omega l}\right|^{2}=1$ and $\left|\mathcal{R}_{\omega l}^{\leftarrow}\right|^{2}$ $+\left|\mathcal{T}_{\omega l}\right|^{2}=1$. The normalization constants $A_{\omega l}$ and $B_{\omega l}$ can be obtained by imposing Klein-Gordon orthonormality of the modes $\psi_{\omega l}$ and $\psi_{\omega l}^{\leftarrow}$ with respect to the Klein-Gordon inner product (14), where Eq. (47) is used to transform the resulting integrals into surface terms (see [7] for details). Then, by using Eqs. (50) and (51), we obtain $A_{\omega l}=B_{\omega l}=(2 \omega)^{-1}$.

The modes $\psi_{\omega l}$ and $\psi_{\omega l}^{\leftarrow}$ can be obtained numerically for small $\omega$ and different $l$ values by evolving Eq. (47) with the effective potential (41) and the asymptotic forms (50) and (51). The corresponding total response $R^{\mathrm{SdS}}$ can be obtained, then, from Eq. (45). We note that the larger the value of $l$, the higher the barrier of the scattering potential $V_{\mathrm{eff}}^{\mathrm{SdS}}(r)$ [24] and therefore the main contributions come from modes with small $l$. How far we must sum over $l$ in Eq. (45) to obtain a satisfactory numerical result will depend on how close to the black hole horizon the source lies. The closer to the horizon the further over $l$ we must sum.

In Fig. 4, we plot the response $R^{\mathrm{SdS}}$ as a function of the source's proper acceleration $a_{0}$ for various values of $\alpha$. As originally defined, $a_{0}$ is not a one-to-one function of the source's radial coordinate $r_{0}$ [cf. Eq. (8)]. In particular, note from Eq. (8) that $a_{0} \rightarrow \infty$ either when $r \rightarrow r_{h}$ or $r \rightarrow r_{c}$. For the sake of clarity in the result presentation, we circumvent this feature by dropping "|.|"' 's in Eq. (8). This is "compensated" by plotting the absolute value of $R^{\mathrm{SdS}}$. After this procedure, the response rate is kept unchanged but $a_{0}$ acquires negative sign between the "equilibrium" point $r_{e}$ $=\left(M \alpha^{2}\right)^{1 / 3}$ (where $\left.a_{0}=0\right)$ and $r_{c}$, becoming then a one-toone function of $r_{0}$. In special, $a_{0} \rightarrow \infty$ when $r_{0} \rightarrow r_{h}$, but $a_{0}$ $\rightarrow-\infty$ when $r_{0} \rightarrow r_{c}$. Moreover, we have "compactified" the range of the proper acceleration from $(-\infty,+\infty)$ to $(0,1)$ by introducing the variable

$$
\chi\left(a_{0}\right)=\left[1-\tanh \left(\rho a_{0}\right)\right] / 2,
$$

which is a monotonic function of the source's proper acceleration $a_{0}$ (and where $\rho$ is a free parameter fixed by numerical convenience). The graph reveals that $R^{\mathrm{SdS}}\left(a_{0}\right)$ tends to $R^{\text {Sch }}\left(a_{0}\right)=q^{2}\left|a_{0}\right| / 4 \pi^{2}$ as $\alpha \rightarrow \infty$, i.e., the response for a source in $\mathrm{SdS}$ spacetime approaches the one in Schwarzschild spacetime (in the Unruh vacuum) when the cosmological constant goes to zero (provided the source keeps the same proper acceleration). This result would be quite expected if we assumed that $r_{0}$ (rather than $a_{0}$ ) was kept constant in the process. This is so because when the cosmological radius $\alpha$ $\rightarrow \infty$, the geometry approaches Schwarzschild's spacetime and the contribution from the cosmological horizon in Eq. (45) becomes negligible both because $\kappa_{c} \rightarrow 0$ and because the low-energy modes emanating from the cosmological horizon have to "travel a longer distance" through the potential barrier to reach the source. As a result, these modes are mostly scattered back, and contribute very little to the total response. However, the explanation is much more subtle when we consider $\alpha \rightarrow \infty$ with $a_{0}$ fixed. For $0<\chi\left(a_{0}\right)<1 / 2$, the larger the $\alpha$ the more the influence of the black hole overcomes that of the cosmic expansion. Thus, as $\alpha \rightarrow \infty$, the "left half" of the curves in Fig. 4 should indeed converge to Schwarzschild for the same reasons pointed out above. Nevertheless, for $1 / 2$ $<\chi\left(a_{0}\right)<1$ the convergence was not expected a priori because in this region $r_{0}>\left(2 M \alpha^{2}\right)^{1 / 3} \gg 2 M \approx r_{h}$, i.e., the larger the $\alpha$ the more the influence of the cosmic expansion overcomes that of the black hole. Indeed, by neglecting the terms $M / r$ and $M / r^{2}$ in Eqs. (1), (8) and (41), we obtain $f_{\mathrm{SdS}}(r) \approx f_{\mathrm{dS}}(r),\left|a_{\mathrm{SdS}}\right| \approx a_{\mathrm{dS}}$ and $V_{\mathrm{eff}}^{\mathrm{SdS}}(r) \approx V_{\mathrm{eff}}^{\mathrm{dS}}(r)$ (where "|. |" is used here to comply with our convention according to which $a_{\mathrm{SdS}}<0$ in this region). Now, in this region, the zero-energy modes ingoing from the black hole are unable to interact with the source (as confirmed by an explicit numerical calculation omitted here), and thus the modes ingoing from the cosmological horizon dominate in Eq. (45). This indicates, at first sight, that $R^{\mathrm{SdS}}\left(a_{0}\right)$ approaches $R^{\mathrm{dS}}\left(a_{0}\right)$ [see Eq. (36)] rather than $R^{\operatorname{Sch}}\left(a_{0}\right)$. The reason why $R^{\mathrm{SdS}}\left(a_{0}\right)$ also approaches $R^{\mathrm{Sch}}\left(a_{0}\right)$ in this region can be understood by recalling that for large enough $\alpha, R^{\mathrm{dS}}\left(a_{0}\right)$ approaches the response for a uniformly accelerated source in Minkowski spacetime $R^{\mathrm{M}}=q^{2} a_{0} / 4 \pi^{2}$ (see Sec. III), which is in turn equivalent to $R^{\mathrm{Sch}}\left(a_{0}\right)$ [6]. We conclude, thus, that the 


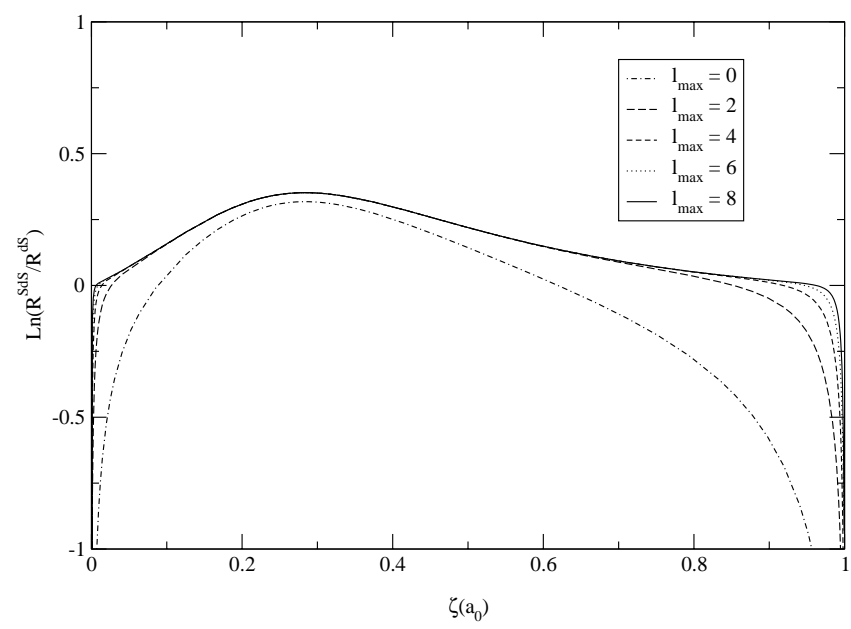

FIG. 5. $\ln \left(R^{\mathrm{SdS}} / R^{\mathrm{dS}}\right)$ is plotted as a function of $\zeta\left(a_{0}\right)$ (with $M$ $=2, \alpha=20$ and $\left.\omega_{0}=10^{-4}\right)$. Here $1_{\max }$ denotes the largest $l$ used in performing the sum (45). $\zeta\left(a_{0}\right)=0$ and $\zeta\left(a_{0}\right)=1$ correspond to the cases where the source is at $r_{0}=r_{h}$ and at $r_{0}=r_{c}$, respectively.

fact that $R^{\mathrm{SdS}}\left(\mathrm{a}_{0}\right) \rightarrow \mathrm{R}^{\mathrm{Sch}}\left(a_{0}\right)$ everywhere when $\alpha \rightarrow \infty$ is a consequence of the (nontrivial) equivalence between the responses in Rindler and Schwarzschild spacetimes.

In Fig. 5 we compare $R^{\mathrm{SdS}}$ with $R^{\mathrm{dS}}$ [cf. Eq. (36)], as a function of $a_{0}$. We use the same convention as in Fig. 4, but now, it is convenient to introduce another compactification variable [25]: $\zeta\left(a_{0}\right)=\left(r_{0}-r_{h}\right) /\left(r_{c}-r_{h}\right)$, where $r_{0}=r_{0}\left(a_{0}\right)$ is the source's radial position as a function of the proper acceleration obtained by inverting Eq. (8) (without "|. |"). Note that, unlike $\chi$, the variable $\zeta$ depends on both $M$ and $\alpha$, which are fixed in Fig. 5. In this figure, the various graphs correspond to the various $1_{\max }$ values of the maximum $l$ used to do the sum (45). Note that the sum converges very fast away from the horizons, but less so for the regions near them. Figure 5 clearly suggests that $R^{\mathrm{SdS}}$ coincides with $R^{\mathrm{dS}}$ near both horizons. That they should coincide near the cosmological horizon could be inferred from our discussion in Fig. 4, but that they coincide in both horizons can be broadly understood from the fact that the $\mathrm{SdS}$ spacetime is isometric to Rindler spacetime near them. This becomes manifest after the change of coordinates $r \mapsto \eta_{h} \equiv\left(2 \kappa_{h}\right)^{-1} \ln \left[f_{\mathrm{SdS}}(r)\right]$ and $r \mapsto \eta_{c} \equiv\left(2 \kappa_{c}\right)^{-1} \ln \left[f_{\mathrm{SdS}}(r)\right]$, which allows one to recast the SdS line element near the black hole and cosmological horizons (apart from the angular piece) as

$$
d s_{\mathrm{SdS}}^{2} \approx e^{2 \kappa_{h} \eta_{h}} d t^{2}-e^{2 \kappa_{h} \eta_{h}} d \eta_{h}^{2}
$$

and

$$
d s_{\mathrm{SdS}}^{2} \approx e^{2 \kappa_{c} \eta_{c}} d t^{2}-e^{2 \kappa_{c} \eta_{c}} d \eta_{c}^{2},
$$

respectively. Now, we recall that the same happens for de Sitter spacetime close to its horizon [cf. Eq. (39)]. As a result, $R^{\mathrm{SdS}}\left(a_{0}\right)$ turns out to approach $R^{\mathrm{dS}}\left(a_{0}\right)$ and both approach $R^{\mathrm{M}}\left(a_{0}\right)=q^{2} a_{0} /\left(4 \pi^{2}\right)$ [cf. Eqs. (36) and (37)] at the horizons.

\section{CONCLUSIONS}

We have quantized here a massless, conformally coupled scalar field in de Sitter and Schwarzschild-de Sitter spacetimes. In both cases, the field interacts with a static scalar source. We have computed the response rates of this source interacting with the Hawking radiation described by the Euclidean vacuum (in the de Sitter case) and by the GibbonsHawking vacuum (in Schwarzschild-de Sitter spacetime). The comparison of the responses (as functions of the proper acceleration of the source) shows, in particular, that the equivalence obtained in [6] between the responses of a static source outside a Schwarzschild black hole (with the Unruh vacuum) and of a uniformly accelerated source in Minkowski spacetime (with the inertial vacuum) was not reproduced here, i.e., the introduction of a cosmological constant breaks the original equivalence.

Although the responses in de Sitter and SdS spacetimes (with the respective vacua) do not coincide, in general, very near the black hole and cosmological horizons they are indeed equivalent. It is so because in both these regions the source is expected to behave as if it were uniformly accelerated in Minkowski spacetime, in the inertial vacuum. We have also recovered everywhere the response of a static source in Schwarzschild spacetime (with the Unruh vacuum) from our response in SdS spacetime as the cosmological constant vanishes. We have shown that this is a direct consequence of the nontrivial equivalence found in [6].

\section{ACKNOWLEDGMENTS}

J.C. and I.S. would like to acknowledge full support from Fundação de Amparo à Pesquisa do Estado de São Paulo (FAPESP). G.M. is thankful to FAPESP and Conselho Nacional de Desenvolvimento Científico e Tecnológico for partial support.
[1] T. Fulton and F. Rohrlich, Ann. Phys. (N.Y.) 9, 499 (1960); F. Rohrlich, Nuovo Cimento 21, 811 (1961); Ann. Phys. (N.Y.) 22, 169 (1963).

[2] D.G. Boulware, Ann. Phys. (N.Y.) 124, 169 (1980).

[3] A. Higuchi, G.E.A. Matsas, and D. Sudarsky, Phys. Rev. D 45, R3308 (1992); 46, 3450 (1992).

[4] S.A. Fulling, Phys. Rev. D 7, 2850 (1973); P.C.W. Davies, J. Phys. A 8, 609 (1975).

[5] W.G. Unruh, Phys. Rev. D 14, 870 (1976).
[6] A. Higuchi, G.E.A. Matsas, and D. Sudarsky, Phys. Rev. D 56, R6071 (1997); 58, 104021 (1998).

[7] J. Castiñeiras and G.E.A. Matsas, Phys. Rev. D 62, 064001 (2000).

[8] L.C.B. Crispino, A. Higuchi, and G.E.A. Matsas, Phys. Rev. D 58, 084027 (1998); 63, 124008 (2001).

[9] J. Castiñeiras, I.P. Costa e Silva, and G.E.A. Matsas, Phys. Rev. D 67, 067502 (2003).

[10] S. Perlmutter et al., Nature (London) 391, 51 (1998); Astro- 
phys. J. 517, 565 (1999); A.G. Riess et al., Astron. J. 116, 1009 (1998).

[11] A.S. Lapedes, J. Math. Phys. 19, 2289 (1978); T. Mishima and A. Nakayama, Phys. Rev. D 37, 348 (1988); A. Nakayama, ibid. 37, 354 (1988); D. Polarski, Class. Quantum Grav. 6, 717 (1989).

[12] N.D. Birrell and P.C.W. Davies, Quantum Field Theory in Curved Space (Cambridge University Press, Cambridge, 1982).

[13] A. Higuchi, Class. Quantum Grav. 4, 721 (1987).

[14] G.W. Gibbons and S.W. Hawking, Phys. Rev. D 15, 2738 (1977).

[15] B.S. Kay and R.M. Wald, Phys. Rep. 207, 49 (1991).

[16] V. Cardoso and J.P.S. Lemos, Phys. Rev. D 67, 084020 (2003).

[17] R.M. Wald, General Relativity (University Press of Chicago, Chicago, 1984).

[18] S.W. Hawking and G.F.R. Ellis, The Large Scale Structure of Space-Time (Cambridge University Press, New York, 1973).
[19] Hereafter, any subscripts (or superscripts) "dS" and "SdS" will designate quantities pertaining to de Sitter and Schwarzschild-de Sitter spacetimes, respectively.

[20] R.M. Wald, Quantum Field Theory in Curved Spacetime and Black Hole Thermodynamics (University Press of Chicago, Chicago, 1994).

[21] D.E. Díaz and J. Stephany, Class. Quantum Grav. 19, 3753 (2002).

[22] I.S. Gradshteyn and I.M. Ryzhik, Table of Integrals, Series and Products (Academic, New York, 1980).

[23] R.C. Tolman, Relativity, Thermodynamics and Cosmology (Claredon, Oxford, 1934).

[24] J. Castiñeiras, L.C.B. Crispino, G.E.A. Matsas, and D.A.T. Vanzella, Phys. Rev. D 65, 104019 (2002).

[25] We emphasize that the use of compactification variables in this paper is entirely for the sake of graphical convenience. All graphs are to be thought of as representing functions of the source's proper acceleration. 